\title{
Non-polynomial spline finite difference method for solving second order boundary value problem
}

\author{
Bushra A. Taha Abdulhassan J. Kadhim \\ Department of Mathematics, Faculty of Science, University of Basrah \\ Basrah, Iraq.
}

Recived : $8 \backslash 3 \backslash 2018$

Revised : $14 \backslash 3 \backslash 2018$

Accepted : 9\4\2018

Available online : $\quad 17 / 5 / 2018$

DOI: $10.29304 / j q c m .2018 .10 .2 .384$

\begin{abstract}
Second -order two-point boundary value problems (BVPs) were solved based on a of nonpolynomial spline general functions with finite difference method.In this paper we discusses a method depended on using special finite-difference approximations for derivatives and formatting a formula that can be deal with endpoints that exceed the usual finite-difference formula for derivatives. Convergence analysis of the method is discussed. The numerical description of the method is shown by four examples. So the results obtained by our method are very encouraging over other existing methods.
\end{abstract}

Keywords: Non-polynomial spline method, Finite difference, Boundary value problem, Truncation error, Exact solution.

\section{Mathematics Classification 65L1265M0641A15.}

\section{Introduction}

In this paper our concern is approximating the solution of the second order linear BVP by using nonpolynomial spline function[5-9,11-13],we discussed the numerical solution for two cases of the problem using non-polynomial spline function.

consider the linear second order BVP of the form $[7,10]$

$$
y^{\prime \prime}(x)+r(x) y^{\prime}+s(x) y=g(x) \quad, x \in[a, b]
$$
subject to the boundary conditions (B.Cs)

$\begin{array}{ll}\text { (A) } \quad y(a)=\alpha_{1} & , y(b)=\beta_{1} \\ \text { (B) } \quad y^{\prime}(a)=\alpha_{2} & , y^{\prime}(b)=\beta_{2}\end{array}$.

Where $r(x), s(x)$ and $g(x)$ are continuous function on $[a, b], \alpha_{1}, \beta_{1}, \alpha_{2}$ and $\beta_{2}$ are real constant .The main objective of our research is to introduce a new spline method to approximate the second order BVP as in (1). 
This paper is organized as follows. In section2, derive of the method with (B.Cs)-(A). Analysis of the method in section 3.In section4, derive of the method with (B.Cs)-(B). In section 5, non-polynomial spline solution.Section 6 convergence analysis .Section 7 some numerical examples to show the performance of the proposed method and for comparison purposes with another numerically methods. Finally, the conclusion is given in section 8 .

The main objective of our research is to introduce a new non-polynomial spline method to approximate the second order boundary value problem as in (1).

\section{2 . Derive of the method with B.Cs -(A)}

We introduced a finite set of grid point $x_{i}$ by dividing the interval $[a, b]$ into $\mathrm{n}$ equal parts where $x_{i}=a+i b, \mathrm{i}=0,1,2, \ldots, \mathrm{n}, \quad x_{0}=a$ $x_{n}=b$ and $h=\frac{b-a}{n+1}$.

Let $y(x)$ be the exact solution of problem (1) and $y_{i}$ be the approximation value to $y\left(x_{i}\right)$ value obtained by the spline function $\varphi_{i}(x)$ passing through the point $\left(x_{i}, y_{i}\right)$ and $\left(x_{i+1}, y_{i+1}\right)$.

Each non-polynomial spline segment has the form [4] $\varphi_{i}(x)=a_{i}+b_{i}\left(x-x_{i}\right)+c_{i} \sinh \left(k\left(x-x_{i}\right)\right)$

$$
\begin{aligned}
& +d_{i} \cosh \left(k\left(x-x_{i}\right)\right) \\
& , i=0,1,2, \ldots, n-1
\end{aligned}
$$

Where $a_{i}, b_{i}, c_{i}, d_{i}$ are constants and $\mathrm{k}$ is free parameter to be determined later. Our non-polynomial spline is now defined by the relations

(i)

$$
y_{i}(x)=\varphi_{i}(x) \quad, x \in\left[x_{i}, x_{i+1}\right] \quad i=1,2, \ldots, n
$$

(ii)

$$
\varphi_{i}(x) \in C^{\infty}\left[x_{i}, x_{i+1}\right] \text {. }
$$

(3)

First, we developd expressions for the four coefficients $a_{i}, b_{i}, c_{i}, d_{i}$ of (2) in terms of $y_{i}, y_{i+1}, \psi_{i}, \psi_{i+1}$,where
(i) $\varphi_{i}\left(x_{i}\right)=y_{i}$
(ii) $\varphi_{i}\left(x_{i+1}\right)=y_{i+1}$
(i) $\varphi_{i}^{\prime \prime}\left(x_{i}\right)=\psi_{i}$
(ii) $\varphi_{i}^{\prime \prime}\left(x_{i+1}\right)=\psi_{i+1}$

(4)
From Eq. (2) and (4), we obtained via a straight forward calculation the following expression: $a_{i}=y_{i}-\frac{h^{2}}{\theta^{2}} \psi_{i}, b_{i}=\frac{1}{h}\left(y_{i+1}-y_{i}\right)+\frac{h}{\theta^{2}}\left(\psi_{i}-\psi_{i+1}\right)$, $d_{i}=\frac{h^{2}}{\theta^{2}} \psi_{i} \quad$ and $\quad c_{i}=\frac{h^{2}}{\theta^{2} \sinh \theta}\left(\psi_{i+1}-\psi_{i} \cosh \theta\right)$ where $i=0,1,2, \ldots, n-1$ and $\theta=k h$.

Now using the continuity of the first derivatives at the point $\left(x_{i}, y_{i}\right)$.that is $\varphi_{i-1}^{\prime}\left(x_{i}\right)=\varphi_{i}^{\prime}\left(x_{i}\right)$ yield the following relations:

$$
y_{i-1}-2 y_{i}+y_{i+1}=h^{2}\left(\xi \psi_{i-1}+2 \eta \psi_{i}+\xi \psi_{i+1}\right)
$$

where

$$
\begin{aligned}
& \psi_{i}=-r\left(x_{i}\right) y^{\prime}-s\left(x_{i}\right) y_{i}+g_{i}\left(x_{i}\right), \\
& \xi=\left(\frac{h}{\theta^{2}}-\frac{h}{\theta \sinh \theta}\right) \text { and } \eta=\left(\frac{-2}{\theta^{2}}+\frac{2 \cosh \theta}{\theta \sinh \theta}\right) .
\end{aligned}
$$

The truncation errors, $t_{i} \quad, i=1,2, \ldots, n$ associated with the scheme (6) can be obtained as follows: first we re-write the scheme (6) in the form

$$
\begin{aligned}
& y_{i-1}-2 y_{i}+y_{i+1}=h^{2}\left[\xi y_{i-1}^{\prime \prime}+2 \eta y_{i}^{\prime \prime}+\xi y_{i+1}^{\prime \prime}\right]+t_{i} \\
& \text { for } i=1,2,3, \ldots, n
\end{aligned}
$$

The terms $y_{i-1}, y_{i}, y_{i+1}$. in Eq.(6)are expanded around the point $x_{i}$ using the Taylor series and the expansion for $t_{i} \quad \mathrm{i}=1,2,3, \ldots . \mathrm{n}$, can be obtained.

$$
t_{i}=\left\{\begin{array}{l}
\left(1-(2 \xi+2 \eta) h^{2} y_{i}^{\prime \prime}+\frac{h^{4}}{12}(1-12 \xi) y_{i}^{(4)}+\right. \\
\frac{24}{360}(1-30 \xi) h^{6} y_{i}^{(6)}+O\left(h^{8}\right) \\
i=1,2,3, \ldots, n .
\end{array}\right\}
$$

The scheme (6) gives rise to family of method of different order as follows:

1 -second order methods

As $k \rightarrow 0 \quad, \xi=\frac{1}{6}$ and $\eta=\frac{1}{3}$.Then 
local truncation error is

$t_{i}=-\frac{1}{12} h^{4} y_{i}^{(4)}+O\left(h^{6}\right) \quad i=1,2, \ldots, n$

2-Forth order methods

For $\xi=\frac{1}{12}$ and $\eta=\frac{5}{12}$.Then the truncation errors in Eq.(6) is

$t_{i}=-\frac{h^{6}}{240} h^{6} y_{i}{ }^{(6)}+O\left(h^{8}\right) \quad i=1,2, \ldots n$

(10)

\section{3 .Analysis of the method with B.Cs.(A)}

To illustrate the application of the spline method developed in the previous section we considered the linear boundary value problem that is given in Eq. (1)at grid point $\left(x_{i}, y_{i}\right)$

we choose an integer $n>0$ and divide the interval $[a, b]$ in $\mathrm{n}$ equal subintervals . Where the step size $h=\frac{b-a}{n+1}$, also approximate forth derivative in Eq.(1) by using non-polynomial spline by substituting $\varphi_{i}=y_{i}{ }^{\prime \prime}$ in Eq.(1) we get the following Eqs.

$\psi_{i-1}=-r\left(x_{i-1}\right) y_{i-1}^{\prime}-s\left(x_{i-1}\right) y_{i-1}+g\left(x_{i-1}\right)$

$\psi_{i}=-r\left(x_{i}\right) y_{i}^{\prime}-s\left(x_{i}\right) y_{i}+g\left(x_{i}\right)$

$\psi_{i+1}=-r\left(x_{i+1}\right) y_{i+1}^{\prime}-s\left(x_{i+1}\right) y_{i+1}+g\left(x_{i+1}\right)$

The first derivative approximate by using finite difference as follow[4]:

$y_{i}^{\prime}=\frac{y_{i+1}-y_{i-1}}{2 h} \quad, \quad y_{i-1}^{\prime}=\frac{-3 y_{i-1}+4 y_{i}-y_{i+1}}{2 h}$

$y_{i+1}^{\prime}=\frac{y_{i-1}-4 y_{i}+3 y_{i+1}}{2 h}$

So Eqs.(11)-(13) and using (14) are become in the form:
Bushra .A/ Abdulhassan .J

$$
\begin{aligned}
& \psi_{i-1}=\frac{-r\left(x_{i-1}\right)\left(-3 y_{i-1}+4 y_{i}-y_{i+1}\right)}{2 h}-s\left(x_{i-1}\right) y_{i-1}+g\left(x_{i-1}\right) . \\
& \psi_{i}=\frac{-r\left(x_{i}\right)\left(y_{i+1}-y_{i-1}\right)}{2 h}-s\left(x_{i}\right) y_{i}+g\left(x_{i}\right) . \\
& \psi_{i+1}=\frac{-r\left(x_{i+1}\right)\left(y_{i-1}-4 y_{i}+3 y_{i+1}\right)}{2 h}-s\left(x_{i+1}\right) y_{i+1}+g\left(x_{i+1}\right) .
\end{aligned}
$$

Now the substituting of the Eq. (15) in (5) we get the following $\mathrm{n}$ linear algebraic Eqs. with $\mathrm{n}$ unknown

$$
\begin{aligned}
& {\left[-1+\xi h\left(\frac{3 r\left(x_{i-1}\right)}{2 h}-\frac{r\left(x_{i+1}\right)}{2}-h^{2} s\left(x_{i-1}\right)\right)+\eta h r\left(x_{i}\right)\right] y_{i-1}} \\
& +\left[2-2 \xi h\left(r\left(x_{i-1}\right)-r\left(x_{i+1}\right)\right)-2 \eta h^{2} s\left(x_{i}\right)\right] y_{i} \\
& \left.\left[-1+\xi h\left(\frac{r\left(x_{i-1}\right)}{h}-\frac{3 r\left(x_{i+1}\right)}{2}\right)-h^{2} s\left(x_{i+1}\right)\right)-\eta h r\left(x_{i}\right)\right] y_{i+1} \\
& =-h^{2} \xi\left(r\left(x_{i-1}\right)+r\left(x_{i+1}\right)\right)-2 h^{2} \eta r\left(x_{i}\right) \quad \text { for } i=1,2, \ldots, n
\end{aligned}
$$

\section{Derive the method with B.Cs-(B)}

Proceeding like in section(2), but we needed to derive special formula when $i=1, n$ using the boundary conditions, finite difference (14) and the step size $h=\frac{b-a}{n+1}$

as

following:

$\left[-\frac{2}{3}+\xi h^{2}\left(\frac{-4 r\left(x_{2}\right)}{3 h}+\frac{4 s\left(x_{0}\right)}{3}\right)+2 \eta h^{2}\left(\frac{2 r\left(x_{1}\right)}{3}+s\left(x_{1}\right)\right)\right] y_{1}$

$+\left[\frac{2}{3}+\xi h^{2}\left(\frac{4 r\left(x_{2}\right)}{3 h}-\frac{s\left(x_{0}\right)}{3}+s\left(x_{2}\right)\right)+4 \eta h^{2} \frac{r\left(x_{1}\right)}{3}\right] y_{2}$

$=\left[\frac{2 h}{3}+\xi h^{2}\left(\frac{r\left(x_{2}\right)}{3}-r\left(x_{0}\right)+\frac{2 h s\left(x_{0}\right)}{3}\right)-\eta h^{2} \frac{r\left(x_{1}\right)}{3}\right] \alpha_{3}$

$+h^{2} \xi\left(r\left(x_{0}\right)+r\left(x_{2}\right)\right)+2 h^{2} \eta r\left(x_{1}\right)$

for $i=1$ 


$$
\begin{aligned}
& \text { [2 } \quad G=\left(g\left(x_{1}\right), g\left(x_{2}\right), \ldots, g\left(x_{n}\right)\right)^{T} \text {, } \\
& {\left[\frac{2}{3}+\xi h^{2}\left(\frac{-4 r\left(x_{n-1}\right)}{3 h}-\frac{s\left(x_{0}\right)}{3}+s\left(x_{n-1}\right)\right)-2 \eta h^{2} \frac{r\left(x_{n}\right)}{3}\right] y_{n-1} \quad Y=\left(y_{1} y_{2}, \ldots, y_{n}\right)^{T}} \\
& +\left[\frac{-2}{3}+\xi h^{2}\left(\frac{4 r\left(x_{n-1}\right)}{3 h}-\frac{4 s\left(x_{n+1}\right)}{3}\right)+2 \eta h^{2}\left(\frac{2 r\left(x_{n}\right)}{3 h}+s\left(x_{n}\right)\right)\right] y_{n} \\
& =\left[\frac{-2 h}{3}+\xi h^{2}\left(\frac{r\left(x_{n-1}\right)}{3}-r\left(x_{n+1}\right)-\frac{2 h s\left(x_{n+1}\right)}{3}\right)-\eta h^{2} \frac{r\left(x_{n}\right)}{3}\right] \alpha_{4}^{D}= \\
& +h^{2} \xi\left(r\left(x_{n-1}\right)+r\left(x_{n+1}\right)\right)+2 h^{2} \eta r\left(x_{n}\right) \\
& \text { for } i=n \text {. } \\
& \text { and } Q=\left(q_{1}, q_{2}, \ldots, q_{n}\right)^{T}
\end{aligned}
$$

\section{Non-polynomial spline solutions}

The scheme (16) gives rise to a linear system of order $(n \times n)$ and may be written in the matrix form as

$$
A y+h^{2} D G=Q
$$

Where $A=C+h B r-h^{2} B s$,

$$
C=\left[\begin{array}{cccccc}
2 & -1 & & & \\
-1 & 2 & -1 & & \\
& -1 & 2 & -1 & \\
& & & \ddots & \\
& & & -1 & 2
\end{array}\right] \text {, }
$$

and $B r=Z_{i j}, B s=U_{i j}$ are define as

$$
\begin{aligned}
M_{i j}=\left\{\begin{array}{l}
-2 \xi\left(r\left(x_{0}\right)-r\left(x_{2}\right)\right) \quad i=1, j=1, \\
\xi\left(\frac{3 r\left(x_{i-1}\right)}{2}-\frac{r\left(x_{i+1}\right)}{2}\right)-h \eta r\left(x_{i}\right) \quad i>j, \\
-2 \xi\left(r\left(x_{i-1}\right)-r\left(x_{i+1}\right)\right) \quad i=j, \\
\xi\left(\frac{r\left(x_{i-1}\right)}{2}-\frac{r\left(x_{i+1}\right)}{2}\right)-h \eta r\left(x_{i}\right) \quad i<j, \\
-2 \xi\left(r\left(x_{n-2}\right)-r\left(x_{n}\right)\right) \quad i=j=n-1,
\end{array}\right. \\
N_{i j}= \begin{cases}2 \eta s\left(x_{i}\right), & i=j=1,2, \ldots, n-1 \\
\xi s\left(x_{i-1}\right), & i>j, \\
\xi s\left(x_{i+1}\right), & i<j\end{cases}
\end{aligned}
$$

where

$\left.q_{1}=-h^{2} \xi r\left(x_{0}\right)-\left(-1+h \xi\left(\frac{3 r\left(x_{0}\right)}{2}+\frac{r\left(x_{0}\right)}{2}\right)-h s\left(x_{0}\right)\right)+h \eta r\left(x_{1}\right)\right) y_{0}$

$q_{i}=0 \quad, i=2,3, \ldots, n-2$.

$q_{n-1}=-h^{2} \xi r\left(x_{n}\right)-\left(-1+h \xi\left(\frac{r\left(x_{n-2}\right)}{2}-\frac{3 r\left(x_{n}\right)}{2}\right)-\right.$

$\left.\left.h s\left(x_{n-2}\right)\right)+h \eta r\left(x_{n-1}\right)\right) y_{n}$

we assume that

$\bar{Y}=\left(y\left(x_{1}\right), y\left(x_{2}\right), \ldots, y\left(x_{n-1}\right)\right)^{T}$.

Be the exact solution of the given boundary value problem (1) at nodal point

$x_{i} \quad$ For $\quad i=1,2, \ldots, n$.then we have

$$
\begin{aligned}
& A \bar{Y}+h^{2} D G=T(h)+Q, \\
& A(\bar{Y}-Y)=A E=T(h)
\end{aligned}
$$

\section{Convergence analysis}

In this section we discuss the convergence property of (6),we now turn back to the error eq. in (18) and rewrite it in the form:

$E=A^{-1} T=\left[C+h B r-h^{2} B s\right]^{-1} T=\left[I+C^{-1}\left(h B r-h^{2} B s\right)\right]^{-1} C^{-1} T$

$\|E\|_{\infty} \leq \|\left[I+C^{-1}\left(h B r-h^{2} B s\right)\left\|_{\infty}\right\| C^{-1}\left\|_{\infty}\right\| T \|_{\infty}\right.$

In order to derive the bound on $\|E\|_{\infty}$, the following two lemmas are needed. 
$\operatorname{Lemma(1)}:$ The matrix $\left(C+h B r-h^{2} B s\right)$ is nonsingular if

$$
\|r\|_{\infty}<\frac{8 h \varepsilon}{(a-b)^{2}(8 \xi+2 \eta)} \text { and }\|s\|_{\infty}<\frac{8(1-\varepsilon)}{(a-b)^{2}}
$$

where $0<\varepsilon<1$.

Proof:

Since,

$A=C+h B r-h^{2} B s=\left[I+C^{-1}\left(h B r-h^{2} B s\right)\right] C$ and the matrix $\mathrm{N}$ is nonsingular, so to prove $\mathrm{A}$ nonsingular it's sufficient to show
$\left[I+C^{-1}\left(h B r-h^{2} B s\right)\right]$ nonsingular.

$$
\begin{aligned}
& \text { Since } \\
& \left\|C^{-1}\left(h B r-h^{2} B s\right)\right\|_{\infty} \leq\left\|C^{-1}\right\|_{\infty}\left\|h B r-h^{2} B s\right\|_{\infty} \\
& \left\|C^{-1}\right\|_{\infty}\left(\|h B r\|_{\infty}+\left\|h^{2} B s\right\|_{\infty}\right)
\end{aligned}
$$

Moreover,

$\left\|C^{-1}\right\|_{\infty} \leq \frac{(a-b)^{2}}{8 h^{2}}[4,10]$ $\|h B r\|_{\infty} \leq h(8 \xi+2 \eta)\|r\|_{\infty}$, and $\left\|h^{2} B s\right\|_{\infty} \leq \begin{gathered}\text { and } \quad l e t y\left(x_{i}\right), i=1,2, \ldots, n-1, \text { satisfies the } \\ e_{i}=y\left(x_{i}\right)-y_{i} \text { then }\end{gathered}$

Where $\|r\|_{\infty}=\max _{a \leq x_{i} \leq b}\left|r\left(x_{i}\right)\right|$, and $\|s\|_{\infty}=\left.\max _{a \leq x_{\text {méth }} \mid}\right|_{\text {od. }}\left(\|_{\infty}^{\infty} x_{i}\right) \mid$

Also from eq. (9) we have:

$\left\|T_{i}\right\|_{\infty}=\frac{h^{6}}{240} F_{6}, F_{6}=\max _{a \leq x \leq b}\left|y^{(6)}\left(x_{i}\right)\right|$.

then

$\leq\|E\|_{\infty} \leq \frac{\left\|C^{-1}\right\|_{\infty}\|T\|_{\infty}}{1-\left\|C^{-1}\right\|_{\infty}\left\|\left(h B r-h^{2} B s\right)\right\|} \cong O\left(h^{4}\right)$

Theorem (1) [3]:

Let $y(x)$ is the exact solution of the continuous boundary value problem (1) with the B.Cs-(A) and (B)

1- $\left\|E_{S}\right\|_{\infty} \cong O\left(h^{2}\right)$ for second order convergent

2- $\|E\|_{\infty} \cong O\left(h^{4}\right)$ for forth order convergent substituting $\|h B r\|_{\infty},\|C\|_{\infty}$, and $\|h B s\|_{\infty}$ in Eq. (23) we get,

$\left\|C^{-1}\left(h B r-h^{2} B s\right)\right\|_{\infty} \leq \frac{(b-a)^{2}}{8 h}(8 \xi+2 \eta)\|r\|_{\infty}$

since

$\|r\|_{\infty}<\frac{8 h \varepsilon}{(b-a)^{2}(8 \xi+2 \eta)}$ and $\|s\|_{\infty}<\frac{8(1-\varepsilon)}{(b-a)^{2}}$

Eq. (23) lead to $\left\|C^{-1}\left(h B r-h^{2} B s\right)\right\|_{\infty} \leq 1$.

from lemma (1), show that the matrix $\mathrm{A}$ is nonsingular.

Since $\left\|C^{-1}\left(h B r-h^{2} B s\right)\right\|_{\infty} \leq 1$. so using

lemma (1) and Eq. (22) we obtine

$\|E\|_{\infty} \leq \frac{\left\|C^{-1}\right\|_{\infty}\|T\|_{\infty}}{1-\left\|C^{-1}\right\|_{\infty}\left\|\left(h B r-h^{2} B s\right)\right\|}$ method.

Whose $\|E\|^{A_{\infty}}$ given by(25)and(26), respectively peglecting all $\mid \mathbf{s} \phi$ rs due to round off.

\section{8}

\section{Numerical examples}

We now consider four numerical examples to illustrate the comparative performance of non-polynomial spline finite difference method in scheme (6).All calculation are implemented by maple 18. In example (1) andexample (2),we the scheme (6) is being applied to solve this problem for $n=16$ and compared with exact solution in tables (1) and( 2 )respectively, moreover for $\mathrm{n}=8,16,32,64$ and 128 . We are compute solutions at grid point, the observed maximum absolute errors $L_{\infty}=\left|y_{i}-y\left(x_{i}\right)\right|$ where $y_{i}$ is the numerical solution and $y\left(x_{i}\right)$ is exact solution all are tabulated in tables (3), (4) respectively, In table (5) our results with the results given in [1] are compared . 
Bushra.A/ Abdulhassan .J

We deduce that our result are more accurate, The figures (1),(2) and(3) illustrate the comparison of the real solution with numerical solution.

In example (3) and example (4), We applied the scheme (6)with Eq.(17)and Eq. (18) to solve this problem for different values $\mathrm{n}$ and compared with exact solution in tables (6) and (7) , moreover $n=8,16,32,64$ and 128, the observed maximumabsolute errors are tabulated in tables (8) and (9), the figures (3) and (4) illustrate the comparison of the real solution with numerical solution.

Example 1 [1]:Consider the boundary value problem

$$
\begin{aligned}
& y^{\prime \prime}-(x+1) y^{\prime}-2 y=\left(1-x^{2}\right) e^{-x} \quad 0 \leq x \leq 1 \\
& y(0)=-1, y(1)=0 .
\end{aligned}
$$

With exact solution

$$
y(x)=(x-1) e^{-x}
$$

Example 2 [1]:Consider the boundary value problem

$$
\begin{aligned}
& y^{\prime \prime}-y^{\prime}=-e^{x-1}-1 \quad 0 \leq x \leq 1 \\
& y(0)=0, y(1)=0 .
\end{aligned}
$$

With exact solution $y(x)=x\left(1-e^{x-1}\right)$.

Example 3 [11]: Consider the boundary value problem

$$
\begin{array}{ll}
y^{\prime \prime}+y=-1 & 0 \leq x \leq 1 \\
y^{\prime}(0)=\frac{1-\cos (1)}{\sin (1)}=-y^{\prime}(1) . &
\end{array}
$$

With exact solution

$$
y(x)=\cos x \frac{1-\cos (1)}{\sin (1)} \sin x-1 \text {. }
$$

Example 4 [9]:Consider the boundary value problem

$$
\begin{aligned}
& -y^{\prime \prime}=\left(2-4 x^{2}\right) y \quad 0 \leq x \leq 1 \\
& y^{\prime}(0)=0, y^{\prime}(1)=\frac{-2}{e^{1}} .
\end{aligned}
$$

With exact solution $y(x)=e^{-x^{2}}$.
Table 1: Comparison numerical solution of nonpolynomial spline finite difference method for $(n=16)$ with exact solution of example (1).

\begin{tabular}{|c|c|c|c|}
\hline \multirow{2}{*}{$\mathrm{n}$} & \multicolumn{2}{|c|}{ numerical solution } & \\
\cline { 2 - 3 } & $\begin{array}{c}\text { Second order } \\
\text { method( } \xi=1 / 6 \\
\eta=1 / 3)\end{array}$ & $\begin{array}{c}\text { Forth order } \\
\text { method } \\
(\xi=1 / 12 \\
\eta=5 / 12)\end{array}$ & exact solution \\
\hline 0.0625 & -0.880669135 & -0.880668474 & -0.880699746 \\
\hline 0.1250 & -0.772131021 & -0.772128861 & -0.77218479 \\
\hline 0.1875 & -0.673515692 & -0.673511558 & -0.673586159 \\
\hline 0.2500 & -0.584018988 & -0.584012695 & -0.584100587 \\
\hline 0.3125 & -0.50289777 & -0.502889373 & -0.502985745 \\
\hline 0.3750 & -0.429465486 & -0.429455222 & -0.429555799 \\
\hline 0.4375 & -0.363088043 & -0.363076287 & -0.363177296 \\
\hline 0.5000 & -0.303179969 & -0.303167199 & -0.30326533 \\
\hline 0.5625 & -0.249200851 & -0.249187614 & -0.249279986 \\
\hline 0.6250 & -0.200652029 & -0.200638912 & -0.200723036 \\
\hline 0.6875 & -0.157073513 & -0.157061126 & -0.157134868 \\
\hline 0.7500 & -0.118041136 & -0.118030087 & -0.118091638 \\
\hline 0.8125 & -0.083163896 & -0.083154783 & -0.083202621 \\
\hline 0.8750 & -0.052081495 & -0.052074892 & -0.052107752 \\
\hline 0.9375 & -0.024462057 & -0.024458503 & -0.024475352 \\
\hline & & & \\
\hline
\end{tabular}

Table 2: Comparison numerical solution of nonpolynomial spline finite difference method for $(n=16)$ with exact solution of example (2).

\begin{tabular}{|c|c|c|c|}
\hline \multirow{2}{*}{$\mathrm{x}$} & \multicolumn{2}{|c|}{ numerical solution } & \multirow{2}{*}{$\begin{array}{c}\text { exact } \\
\text { method( } \xi=1 / 6 \\
\eta=1 / 3)\end{array}$} \\
\cline { 2 - 3 } & $\begin{array}{c}\text { Forth order } \\
\text { method } \\
(\xi=1 / 12 \\
\eta=5 / 12)\end{array}$ & \\
\hline 0.0625 & 0.038043273 & 0.038039019 & 0.038024648 \\
\hline 0.1250 & 0.072928609 & 0.072920342 & 0.072892248 \\
\hline 0.1875 & 0.104350364 & 0.104338372 & 0.104297379 \\
\hline 0.2500 & 0.131976602 & 0.131961227 & 0.131908362 \\
\hline 0.3125 & 0.155449676 & 0.155428619 & 0.155365132 \\
\hline 0.3750 & 0.174370442 & 0.174349572 & 0.174276964 \\
\hline 0.4375 & 0.188322801 & 0.188299959 & 0.188220014 \\
\hline 0.5000 & 0.196844046 & 0.196819852 & 0.19673467 \\
\hline 0.5625 & 0.199435511 & 0.199410674 & 0.199322704 \\
\hline 0.6250 & 19555679100 & 0.195532119 & 0.195444201 \\
\hline 0.6875 & 0.184622441 & 0.184598846 & 0.184514255 \\
\hline 0.7500 & 0.165998192 & 0.165976919 & 0.165899413 \\
\hline 0.8125 & 0.138998192 & 0.13897997 & 0.138913842 \\
\hline 0.8750 & 0.102878735 & 0.102865077 & 0.10281521 \\
\hline 0.9375 & 0.056835959 & 0.056828319 & 0.056800254 \\
\hline
\end{tabular}


Table 3:The maximum absolute errors ofnonpolynomial spline finite difference method for example (1).

\begin{tabular}{|c|c|c|}
\hline \multirow{2}{*}{$n$} & \multicolumn{2}{|c|}{ max norm(L) } \\
\cline { 2 - 3 } & $\begin{array}{c}\text { Second order } \\
\text { method( } \xi=1 / 6, \\
\eta=1 / 3)\end{array}$ & $\begin{array}{c}\text { Forth order } \\
\text { method } \\
(\xi=1 / 12 \\
\eta=5 / 12)\end{array}$ \\
\hline 8 & $3.63 \mathrm{E}-04$ & $4.04 \mathrm{E}-04$ \\
\hline 16 & $9.03129 \mathrm{E}-05$ & $1.01 \mathrm{E}-04$ \\
\hline 32 & $2.25 \mathrm{E}-05$ & $2.53 \mathrm{E}-05$ \\
\hline 64 & $5.57478 \mathrm{E}-06$ & $6.26843 \mathrm{E}-06$ \\
\hline 128 & $9.87 \mathrm{E}-07$ & $1.2426 \mathrm{E}-06$ \\
\hline
\end{tabular}

Table 4:The maximum absolute errors of nonpolynomial spline finite difference method for example (2).

\begin{tabular}{|c|c|c|}
\hline \multirow{2}{*}{$\mathrm{n}$} & \multicolumn{2}{|c|}{ max norm(L) } \\
\cline { 2 - 3 } & $\begin{array}{c}\text { Second order } \\
\text { method( }(=1 / 6, \\
\eta=1 / 3)\end{array}$ & $\begin{array}{c}\text { Forth order } \\
\text { method } \\
(\xi=1 / 12 \\
\eta=5 / 12)\end{array}$ \\
\hline 8 & 0.00045128 & 0.000352401 \\
\hline 16 & $1.12 \mathrm{E}-04$ & $8.79708 \mathrm{E}-05$ \\
\hline 32 & $2.83 \mathrm{E}-05$ & $2.20701 \mathrm{E}-05$ \\
\hline 64 & $7.06974 \mathrm{E}-06$ & $5.51684 \mathrm{E}-06$ \\
\hline 128 & $1.76738 \mathrm{E}-06$ & $1.38 \mathrm{E}-06$ \\
\hline
\end{tabular}

Table 5:Comparison the maximum absolute errors ofnon-polynomial spline finite difference method for example (1) and example (2) for $(n=32)$ with the maximum absolute errors of B-spline method[1].

\begin{tabular}{|c|c|c|c|}
\hline \multicolumn{4}{|c|}{ maximum error } \\
\hline \multirow{4}{*}{} & \multicolumn{2}{|c|}{$\begin{array}{c}\text { non-polynomial spline } \\
\text { finite difference method }\end{array}$} \\
\cline { 2 - 4 } & $\begin{array}{c}\xi=1 / 6, \\
\eta=1 / 3\end{array}$ & $\begin{array}{c}\xi=1 / 12 \\
\eta=5 / 12\end{array}$ & $\begin{array}{c}\text { B-spline } \\
\text { method [1] }\end{array}$ \\
\hline $\begin{array}{c}\text { example } \\
1\end{array}$ & $2.25 \mathrm{E}-05$ & $2.53 \mathrm{E}-05$ & $5.70 \mathrm{E}-04$ \\
\hline $\begin{array}{c}\text { example } \\
2\end{array}$ & $2.83 \mathrm{E}-05$ & $2.27 \mathrm{E}-05$ & $6.88 \mathrm{E}-04$ \\
\hline
\end{tabular}

Table 6:Comparison numerical solution of nonpolynomial spline finite difference method for $(n=16)$ with exact solution of example (3).

\begin{tabular}{|c|c|c|c|}
\hline \multirow[b]{2}{*}{$\mathrm{x}$} & \multicolumn{2}{|c|}{ numerical solution } & \multirow[b]{2}{*}{$\begin{array}{c}\text { exact } \\
\text { solution }\end{array}$} \\
\hline & $\begin{array}{c}\text { Second order } \\
\operatorname{method}(\xi=1 / 6 \\
\eta=1 / 3)\end{array}$ & $\begin{array}{l}\text { Forth order } \\
\text { method } \\
(\xi=1 / 12 \\
\eta=5 / 12)\end{array}$ & \\
\hline 0.0625 & 0.031299775 & 0.030943688 & 0.032169192 \\
\hline 0.1250 & 0.05940484 & 0.059048874 & 0.060307784 \\
\hline 0.1875 & 0.083374298 & 0.083018496 & 0.084305898 \\
\hline 0.2500 & 0.103114577 & 0.102758954 & 0.104069821 \\
\hline 0.3125 & 0.118548619 & 0.118193162 & 0.119522374 \\
\hline 0.3750 & 0.129616173 & 0.129260849 & 0.130603217 \\
\hline 0.4375 & 0.136274035 & 0.159187958 & 0.137269078 \\
\hline 0.5000 & 0.138496213 & 0.138141004 & 0.139493927 \\
\hline 0.5625 & 0.136274035 & 0.135918796 & 0.137269078 \\
\hline 0.6250 & 0.129616173 & 0.129260849 & 0.130603217 \\
\hline 0.6875 & 0.118548619 & 0.118193162 & 0.119522374 \\
\hline 0.7500 & 0.103114577 & 0.102758954 & 0.104069821 \\
\hline 0.8125 & 0.083372975 & 0.083018496 & 0.084305898 \\
\hline 0.8750 & 0.05940484 & 0.059048874 & 0.060307785 \\
\hline 0.9375 & 0.031299775 & 0.030943688 & 0.032169192 \\
\hline
\end{tabular}

Table 7:Comparison numerical solution of nonpolynomial spline finite difference method for $(n=16)$ with exact solution of example (4).

\begin{tabular}{|c|c|c|c|}
\hline \multirow[b]{2}{*}{$\mathrm{x}$} & \multicolumn{2}{|c|}{ numerical solution } & \multirow[b]{2}{*}{ exact solution } \\
\hline & $\begin{array}{c}\text { Second order } \\
\operatorname{method}(\xi=1 / 6 \\
\eta=1 / 3)\end{array}$ & $\begin{array}{c}\text { Forth order } \\
\text { method } \\
(\xi=1 / 12, \\
\eta=5 / 12)\end{array}$ & \\
\hline 0.0625 & 0.994624959 & 0.993898323 & 0.99610137 \\
\hline 0.1250 & 0.983104909 & 0.982364394 & 0.984496437 \\
\hline 0.1875 & 0.964172404 & 0.963409595 & 0.965454552 \\
\hline 0.2500 & 0.938262193 & 0.937469997 & 0.939413063 \\
\hline 0.3125 & 0.905959649 & 0.905132689 & 0.906960618 \\
\hline 0.3750 & 0.867978854 & 0.867113733 & 0.868815056 \\
\hline 0.4375 & 0.825136448 & 0.824231864 & 0.82579704 \\
\hline 0.5000 & 0.778322587 & 0.777379302 & 0.778800783 \\
\hline 0.5625 & 0.728470452 & 0.72749111 & 0.72876333 \\
\hline 0.6250 & 0.676525748 & 0.675514562 & 0.676633846 \\
\hline 0.6875 & 0.623417583 & 0.622379908 & 0.623344309 \\
\hline 0.7500 & 0.570031932 & 0.568973763 & 0.569782825 \\
\hline 0.8125 & 0.517188709 & 0.51611612 & 0.516770583 \\
\hline 0.8750 & 0.465623173 & 0.464541742 & 0.465043188 \\
\hline 0.9375 & 0.415972143 & 0.414886376 & 0.415236829 \\
\hline
\end{tabular}


Table 8:The maximum absolute errors of nonpolynomial spline finite difference method for example (3).

\begin{tabular}{|c|c|c|}
\hline \multirow{2}{*}{$\mathrm{N}$} & \multicolumn{2}{|c|}{ Maximum error } \\
\cline { 2 - 3 } & $\begin{array}{c}\text { Second order } \\
\text { method( } \xi=1 / 6 \\
\eta=1 / 3)\end{array}$ & $\begin{array}{c}\text { Forth order method } \\
(\xi=1 / 12 \\
\eta=5 / 12)\end{array}$ \\
\hline 8 & $3.45 \mathrm{E}-03$ & $4.87 \mathrm{E}-03$ \\
\hline 16 & $9.97 \mathrm{E}-04$ & $1.35 \mathrm{E}-03$ \\
\hline 32 & $2.66 \mathrm{E}-04$ & $3.55 \mathrm{E}-04$ \\
\hline 64 & $6.82946 \mathrm{E}-05$ & $9.08673 \mathrm{E}-05$ \\
\hline 128 & $1.86 \mathrm{E}-05$ & $2.27 \mathrm{E}-05$ \\
\hline
\end{tabular}

Table 9:The maximum absolute errors of nonpolynomial spline finite difference method for example (4).

\begin{tabular}{|c|c|c|}
\hline $\mathrm{n}$ & $\begin{array}{c}\text { Second order } \\
\text { method( } \xi=1 / 6, \\
\eta=1 / 3)\end{array}$ & $\begin{array}{c}\text { Forth order } \\
\text { method } \\
(\xi=1 / 12 \\
\eta=5 / 12)\end{array}$ \\
\hline 8 & $7.15 \mathrm{E}-03$ & $7.15 \mathrm{E}-03$ \\
\hline 16 & $1.48 \mathrm{E}-03$ & $2.20 \mathrm{E}-03$ \\
\hline 32 & $4.20 \mathrm{E}-04$ & $6.03 \mathrm{E}-04$ \\
\hline 64 & $1.11 \mathrm{E}-04$ & $3.93 \mathrm{E}-05$ \\
\hline 128 & $2.68 \mathrm{E}-05$ & $3.93377 \mathrm{E}-05$ \\
\hline
\end{tabular}

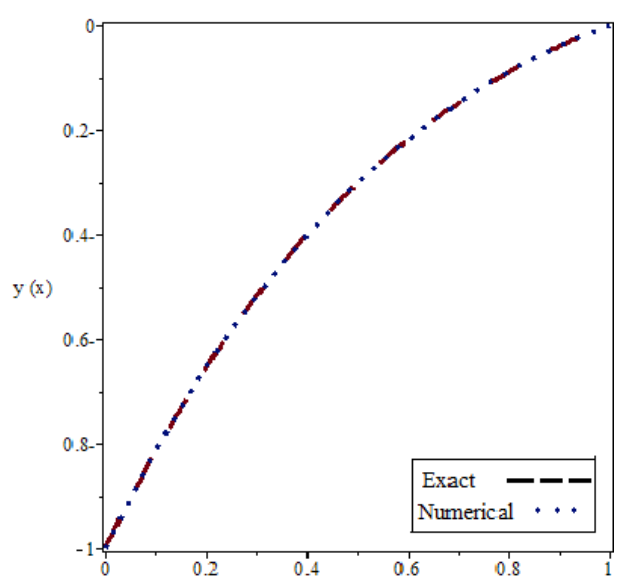

Figure(1):Comparison the exact and numerical solution $(\mathrm{h}=1 / 32)$ for

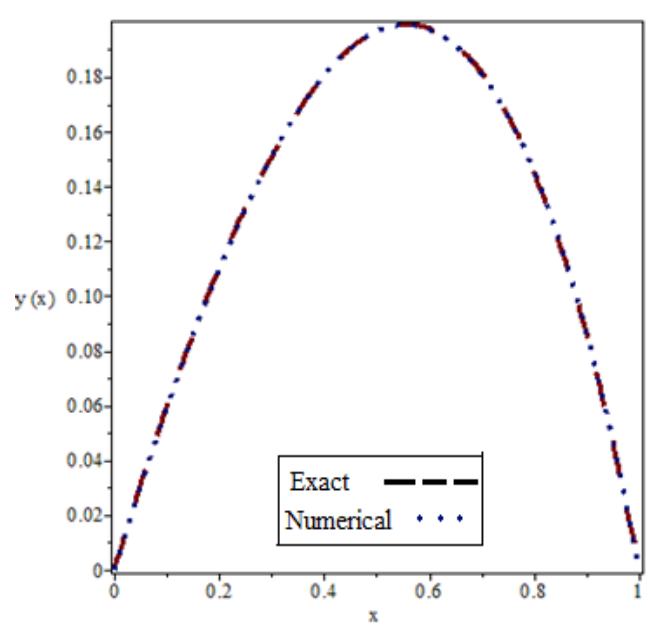

Figure(2): Gomparison the exact and numerical solution $(\mathrm{h}=1 / 32)$ for example( 2$)$.

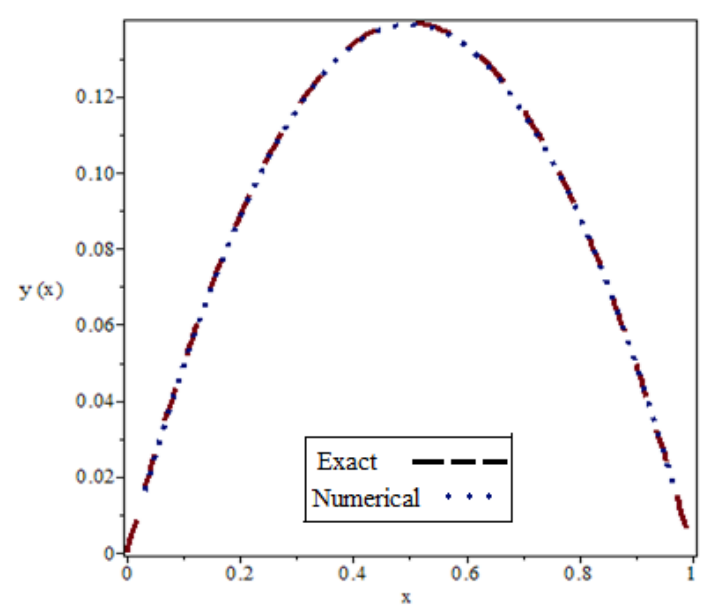

Figure(3):Comparison the exact and numerical solution $(h=1 / 32)$ for example (3).

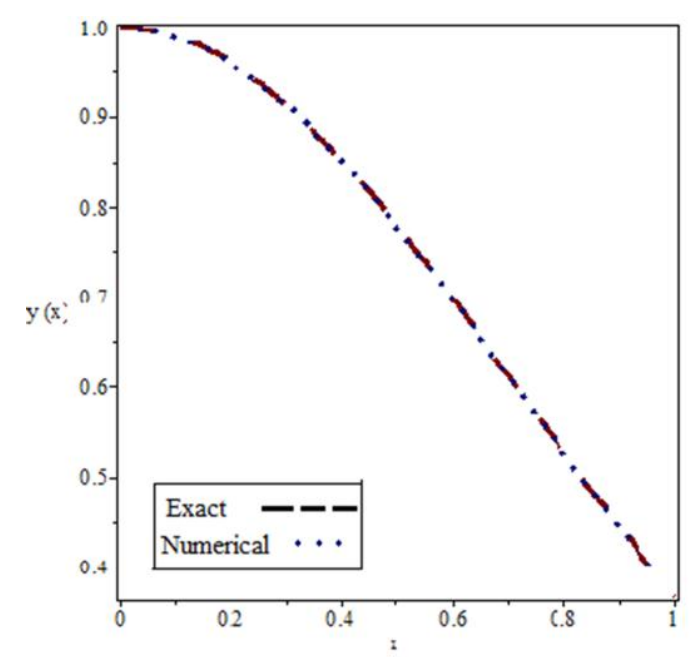

Figure(4):Comparison the exact and numerical solution $(\mathrm{h}=1 / 32)$ for example (4) . 


\section{Conclusion}

In this paper, the non-polynomial spline finite difference method was used to solve the general linear of the second boundary value problem, and show that this method is better in terms of accuracy and application, these have been verified by maximum absolute errors.

\section{References}

[1] N. N. Abd Hamid, A. Abd. Majid, and A. Izani Md. Ismail, Cubic trigonometric B-spline applied to linear two-point boundary value problems of order Two, World Academy of Science, Engineering and Technology International Journal of Mathematical and Computational Sciences Vol:4, No:10, 2010.

[2] B. O. Al-Azzah, Numerical methods for solving higher-order boundary value problems. M.sc Thesis, An-Najah National University, Faculty of Graduate Studies, 1992.

[3] F.I.Haq and S. I. Abbas ,The numerical solution boundary valueand initial- boundary valueproblems usingspline functions, GhulamIshaq Khan Institute of Engineering Sciences and Technology,2009.

[4] R. Jalilian, Convergence analysis of spline solutions for special nonlinear two-order boundary value problems, World Applied Sciences Journal 7 (Special Issue of Applied Math): 19-24, 2009.

[5] N. Jha, An Intermediate Non-polynomial spline algorithm for second order nonlinear differential problems: Applications to Physiology and Thermal Explosion, K.N. Das et al. (eds.), Proceedings of Fourth International Conference on Soft Computing for Problem Solving, Advances in Intelligent Systems and Computing 336, DOI 10.1007/978-81322-2220-0 52,2015.

[6] N.t Jha and R.K. Mohanty, Quintic hyperbolic non-polynomial spline and finite difference method for nonlinear second order differential equations and its application, Journal of the Egyptian Mathematical Society (2014) 22, 115-122.
[7] H. Justine, J.Vui and J. Sulaiman , Quariticnonpolynomialspline solution for solving two-point boundary value problems by using conjugate gradient iterative method, Journal of Applied Mathematics and Computational Mechanics 2017, 16(1), 41-50.

[8] P. Kalyani and P. S. Rama Chandra Rao , Solution of boundary value problems by approaching spline techniques, Hindawi Publishing Corporation International Journal of Engineering Mathematics Volume 2013, Article ID 482050, 9 pages.

[9] M.A. Ramadan , I.F. Lashien and W.K. Zahra, Polynomial and non-polynomial spline approaches to the numerical solution of second order boundary value problems, Applied Mathematics and Computation 184 (2007) 476-484.

[10] J. Rashidinia , R. Jalilian and R. Mohammad, Convergence analysis of spline solution of certain two-point boundary value problems, Computer Science \& Engineering and Electrical Engineering Vol. 16, No. 2, pp. $128\{136,2009$.

[11] P. K. Srivastava , M. Kumar and R.N. Mohapatra, Quintic non-polynomial spline method for the solution of a second-order boundary-value problem with engineering applications, Computers and Mathematics with Applications 62 (2011) 1707-1714.

[12] B. A. Taha and A. R. Khlefha , Numerical solution of third order BVPs by using nonpolynomial spline with FDM , Nonlinear Analysis and Differential Equations, Vol. 3, 2015, no. 1, 1 21.

[13] S.Ul-Eslam and S.Akram, Numerical solution of boundary value problems using non-polynomial spline functions ,Ghulam,Ishaq Institute Of Engineering Science and Tecnology,2005. 


\title{
طريقة الغير متعددات الدود السبلاين مع الفروقات المحدة لحل مسائل قيم حدودية من الرتبة الثانية
}

\author{
بشرى عزيز طله عبد الحسن جواد كاظم

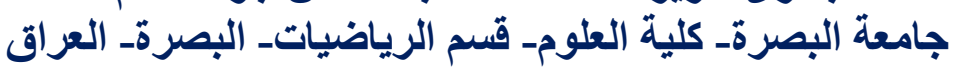

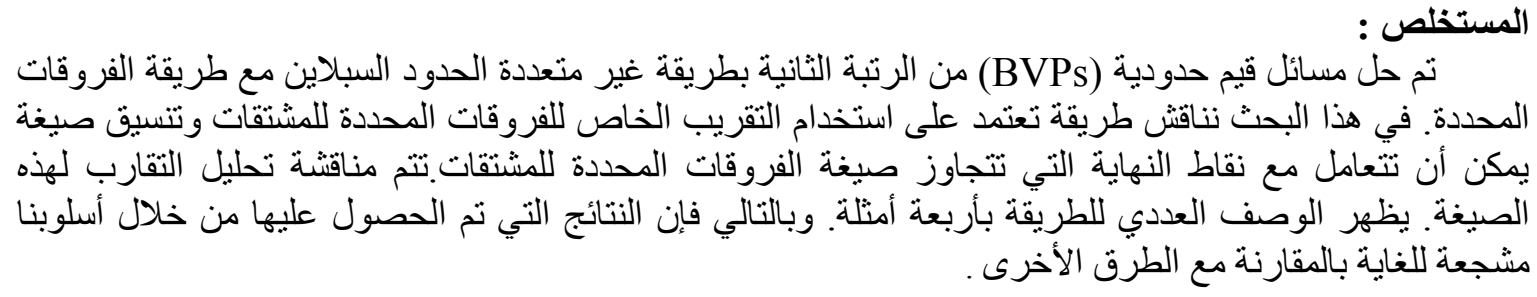

الكلمات المفتاحية: طريقة الغير متعددة الحدود السبلاين ،الفروقات المحددة، مسائل القيم الحدودية، خطأ القطع، الحل

الحقيقي. 